\title{
Enzalutamide Treatment in Metastatic Castration-resistant Prostate Cancer: Before and after Docetaxel
}

\author{
Irem Bilgetekin ${ }^{1}$, Fatma Bugdayci Basal ${ }^{1}$, Havva Yesil Cinkir ${ }^{2}$, Ece Esin ${ }^{1}$, Berna Oksuzoglu ${ }^{1}$ and Umut Demirci ${ }^{1}$ \\ ${ }^{1}$ Department of Medical Oncology, Dr. Abdurrahman Yurtaslan Ankara Oncology Training and Research Hospital, Ankara, Turkey \\ ${ }^{2}$ Department of Medical Oncology, Gaziantep University Medical Faculty Hospital, Gaziantep, Turkey
}

\begin{abstract}
Objective: To evaluate the efficacy and safety of enzalutamide in metastatic castration-resistant prostate cancer (mCRPC) in docetaxel-naive and docetaxel-pretreated patients.

Study Design: Observational study.

Place and Duration of Study: HSU Dr. Abdurrahman Yurtaslan Oncology Training and Research Hospital, Department of Medical Oncology, Ankara, Turkey, from March 2017 to July 2019.

Methodology: A total of 67 patients with $\mathrm{MCRPC}$ were retrospectively evaluated. Castration-naive patients and non-metastatic patients were excluded from the study. Comorbid diseases, ECOG performance status, PSA response, and the radiological response of the patients were recorded. Kaplan-Meier method was used for survival analysis, and a Cox regression model was formed.

Results: The overall survival (OS) was significantly longer in patients with eastern cooperative oncology group performance status (ECOG PS) 0 ( 26.0 vs. 14.0 months, $p=0.031$ ), PSA response $(26.0$ vs. 7.0 months, $p=0.002)$, radiological response $(26.0$ vs. 10.0 months, $p=0.006$ ) and duration of enzalutamide $\geq 9$ months ( 26.0 vs. 7.0 months, $p<0.001$ ) compared to ECOG PS 1 . According to Cox regression analysis, patients with PSA response had 0.35 fold (Cl.95\% $0.13-0.94)$ reduced the risk of death and 0.36 -fold $(\mathrm{Cl} .95 \% 0.16-0.85)$ reduced the risk of progression compared to those without PSA response. Moreover, longer enzalutamide treatment ( $\geq 9$ months) was noted to decrease the risk of death.

Conclusion: PSA response, radiological response and duration of enzalutamide treatment may predict the improvement of survival in patients with $\mathrm{mCRPC}$ treated with enzalutamide.
\end{abstract}

Key Words: Enzalutamide, Docetaxel, Castration-resistant prostate cancer, Overall survival, Progression-free survival.

How to cite this article: Bilgetekin I, Basal FB, Cinkir HY, Esin E, Oksuzoglu B, Demirci U. Enzalutamide Treatment in Metastatic Castration-resistant Prostate Cancer: Before and after Docetaxel. J Coll Physicians Surg Pak 2020; 30(08):815-821.

\section{INTRODUCTION}

Prostate cancer $(\mathrm{PC})$ is the most common male cancer worldwide, accounting for $1-2 \%$ of deaths in men. ${ }^{1}$ Prostate cancer is approximately $5 \%$ metastatic at the time of diagnosis. ${ }^{2}$ Considering the known effects of androgens on the progression of PC, androgen deprivation therapy (ADT) remains the gold standard of treatment for $\mathrm{PC}^{3,4}$ However, castration-resistant prostate cancer (CRPC) recurs within an average of 14-20 months after ADT through castration. ${ }^{5}$

Correspondence to: Dr. Irem Bilgetekin, Department of Medical Oncology, Dr. Abdurrahman Yurtaslan Ankara Oncology Training and Research Hospital, Ankara, Turkey E-mail:irembilgetekin@gmail.com

Received: July 03, 2020; Revised: July 05, 2020;

Accepted: July 09, 2020

DOI: https://doi.org/10.29271/jcpsp.2020.08.815
Castration-resistant means the progression of disease despite a serum testosterone level of $<50 \mathrm{ng} / \mathrm{dl}(<1.7 \mathrm{nmol} / \mathrm{l}) .{ }^{6}$ Castration resistance in prostate cancer increases the risk of metastasis and one-third of patients become metastatic-disease within 2 years. $^{7}$

In metastatic CRPC, there have been significant improvements in treatment with the use of hormonal treatment (enzalutamide or abiraterone acetate with prednisone) agents before cytotoxic chemotherapy. ${ }^{8}$ Until date, various treatment options have been approved for metastatic CRPC (MCRPC), including docetaxel, cabazitaxel, abiraterone acetate, enzalutamide, sipuleucel-T, and radium-223, ${ }^{9}$ after the use of docetaxel cabazitaxel provided survival advantage. ${ }^{10}$

Preclinical studies have evidenced that androgen receptor (AR) overexpression resists ADT in the PC cell line, and intratumoral androgen levels were often noted to be high in patients with mCRPC. ${ }^{11,12}$ With advancements in the understanding of CRPC biology, several targeted treatment strategies have been developed to overcome AR signalling. . $^{13,14}$ 
Table I: Summary of patients' characteristics based on enzalutamide treatment order.

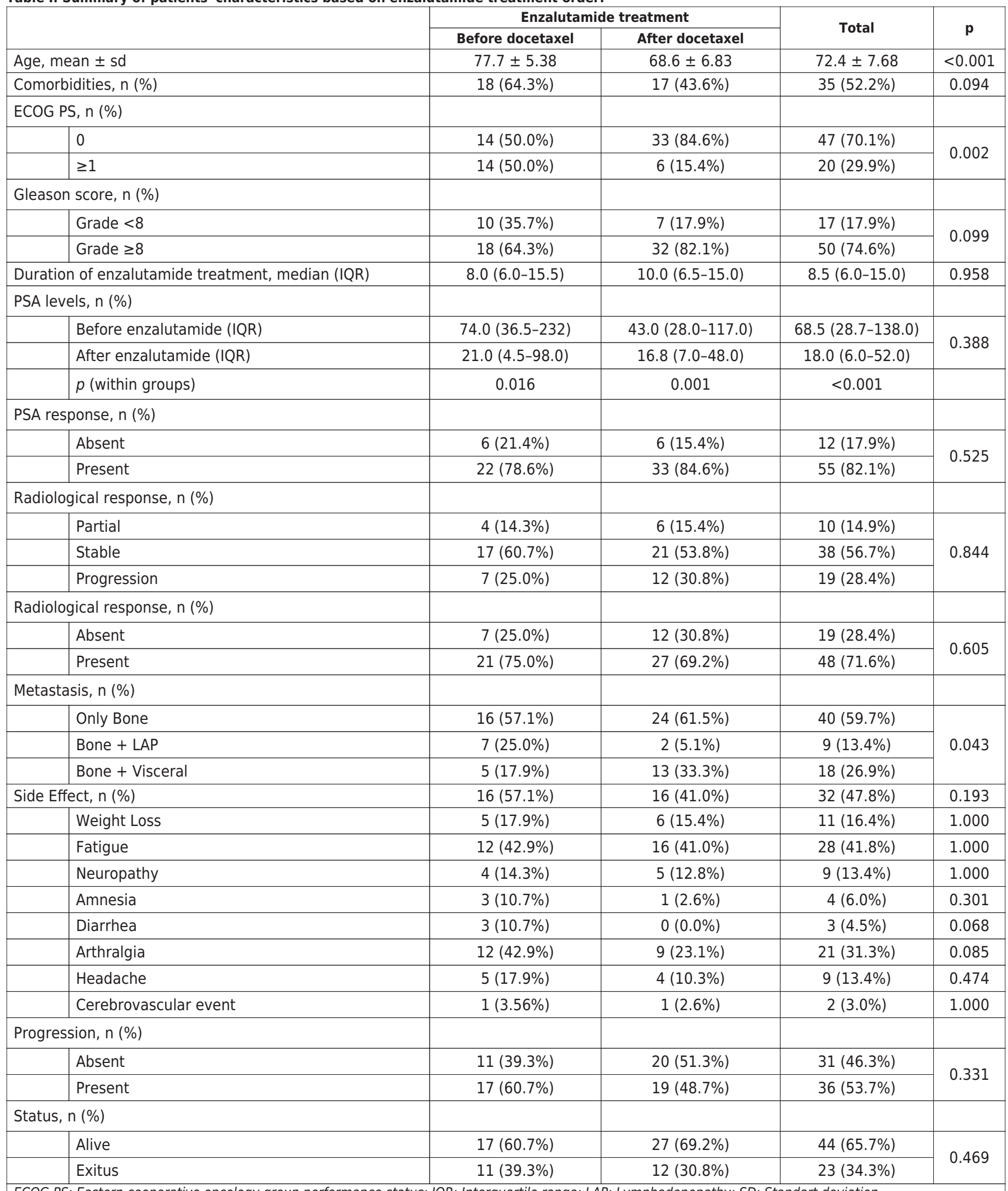

ECOG PS: Eastern cooperative oncology group performance status; IQR: Interquartile range; LAP: Lymphadenopathy; SD: Standart deviation.

Abiraterone and enzalutamide are hormonal treatment agents that improve survival. While abiraterone acts on survival by androgen synthesis blockade, enzalutamide acts by blocking androgen receptor. ${ }^{15}$ Enzalutamide belongs to the class of AR inhibitors that act by first binding directly to the $A R$ and subsequently blocking both AR-mediated DNA 
binding and AR nuclear translocation. ${ }^{16,17}$ The effect of enzalutamide on overall survival (OS) has been demonstrated in the PREVAIL and AFFIRM studies, which are international, placebo-controlled, phase 3 studies involving patients with mCRPC, both docetaxel-naive and docetaxel-pretreated ${ }^{18,19}$

In Turkey, the use of enzalutamide as the first-line treatment is limited only to patients who cannot tolerate docetaxel. Besides, it is most commonly used after docetaxel therapy in routine practice. Hence, this study aimed to evaluate the efficacy and safety of enzalutamide in docetaxel-naive and docetaxel-pretreated patients. To the authors' knowledge, this is the first study to analyse the real-world data from Turkey regarding $\mathrm{MCRPC}$.

\section{METHODOLOGY}

In this retrospective observational study, patients who had radiological evidence for metastasis and were followed up with histologically confirmed $\mathrm{MCRPC}$ from the medical file databases of the two oncology centres were identified. Among these patients, those who had received enzalutamide (as first-line treatment or after docetaxel) for mCRPC between March 2017 and July 2019 were enrolled. The efficacy of enzalutamide treatment in MCRPC, before and after docetaxel, was investigated in the present study.

Patients were included if they had histologically verified adenocarcinoma of the prostate with described metastases, as well as PSA and radiological progressions (or both) in bone or soft tissue metastases, and were considering LHRH analogue therapy or orchiectomy, with a serum testosterone level of $1.73 \mathrm{nmol} / \mathrm{L}(50 \mathrm{ng} / \mathrm{dL}$ ) or less. All patients with MCRPC, who received first-line enzalutamide or began enzalutamide after docetaxel therapy, were included in the study. Castration-naive patients and non-metastatic patients were excluded from the study.

The following parameters were evaluated within the scope of the study: comorbid diseases, ECOG performance status, Gleason score, PSA response, clinical response, radiological response, enzalutamide therapy characteristics (first-line, after docetaxel, other treatments), side effects and toxicity information, metastatic sites, progression status, and exitus dates.

Radiological response was evaluated by thorax, abdomen and pelvis tomography, and include scintigraphy (bone scan) for evaluation of bone metastases.

Enzalutamide was prescribed at a dose of $160 \mathrm{mg} /$ day and was administered until disease progression, death, or development of unacceptable toxicity. Patients who received first line enzalutamide treatment were designated as the "before docetaxel' (BD) group. Patients who received enzalutamide after docetaxel were designated as the 'after docetaxel' (AD) group. All side effects, including possible drug-related fatigue symptoms, as well as all neurological findings, including seizures, were recorded in patients' tolerance and reliability analyses.

All analyses were performed using SPSS version \# 21 (SPSS Inc., Chicago, IL, USA). Repeated measurements of PSA values were compared between time-points by using Wilcoxon T test. Intergroup comparisons of PSA value differences were performed using the Mann-Whitney U-test. Categorical variables were evaluated using the Chi-square tests or Fisher's Exact tests.

The presence of 'PSA response' was defined as more than $50 \%$ reduction from baseline. The radiological response was considered complete or partial in the presence of regression or stable disease.

Survival analyses were performed using Kaplan-Meier method. Intergroup survival comparisons were performed using the Log-Rank test. Notably, the time from the initiation of enzalutamide to progression was defined as progression-free survival (PFS); whereas, the time from the initiation of enzalutamide until death or the last control date was considered as OS. Significant factors of death and progression were determined using the Cox regression analysis forward selection (conditional) method. Two-tailed $p$ values of less than 0.05 were considered statistically significant.

\section{RESULTS}

The number of patients receiving enzalutamide before docetaxel was $28(41.8 \%)$, after docetaxel was 39 (58.2\%). Median duration of enzalutamide treatment was 8.5 months. The mean age of study patients was $72.40 \pm 7.68$ years (range: $53-85$ years), and 35 patients (52.2\%) had comorbidities. The median PSA value at the time of diagnosis was 52 $\mathrm{ng} / \mathrm{ml}$ (IQR: $13-171 \mathrm{ng} / \mathrm{ml}$ ). The BD group was observed to be significantly older $(p<0.001)$ and worse performance score $(p=0.002)$. PSA levels were noted to decrease significantly in all groups (BD: $p=0.016, A D: p=0.001$ ). The summary of patients' characteristics related to enzalutamide treatment initiation is provided in Table I.

OS was significantly higher in patients with PSA response (26.0 vs. 7.0 months, $p=0.002$, Figure 1 ). OS was significantly higher in patients with radiological response (26.0 vs. 10.0 months, $p=0.006)$. Data regarding intergroup OS and PFS comparisons with the Log-Rank test are presented in Table II.

The presence of PSA response and longer duration of enzalutamide treatment ( $\geq 9$ months) were determined to be good prognostic factors for OS upon Cox regression analysis. Patients with PSA response had 0.35 -fold lower likelihood of death [Cl. 95\% (0.13-0.94)]; whereas, longer enzalutamide treatment duration ( $\geq 9$ months) 0.72 -fold decreased the risk of death $[\mathrm{Cl} .95 \%(0.63-0.83)]$. 
Table II: OS and PFS with Kaplan-Meier method and comparisons of groups with Log-Rank test.

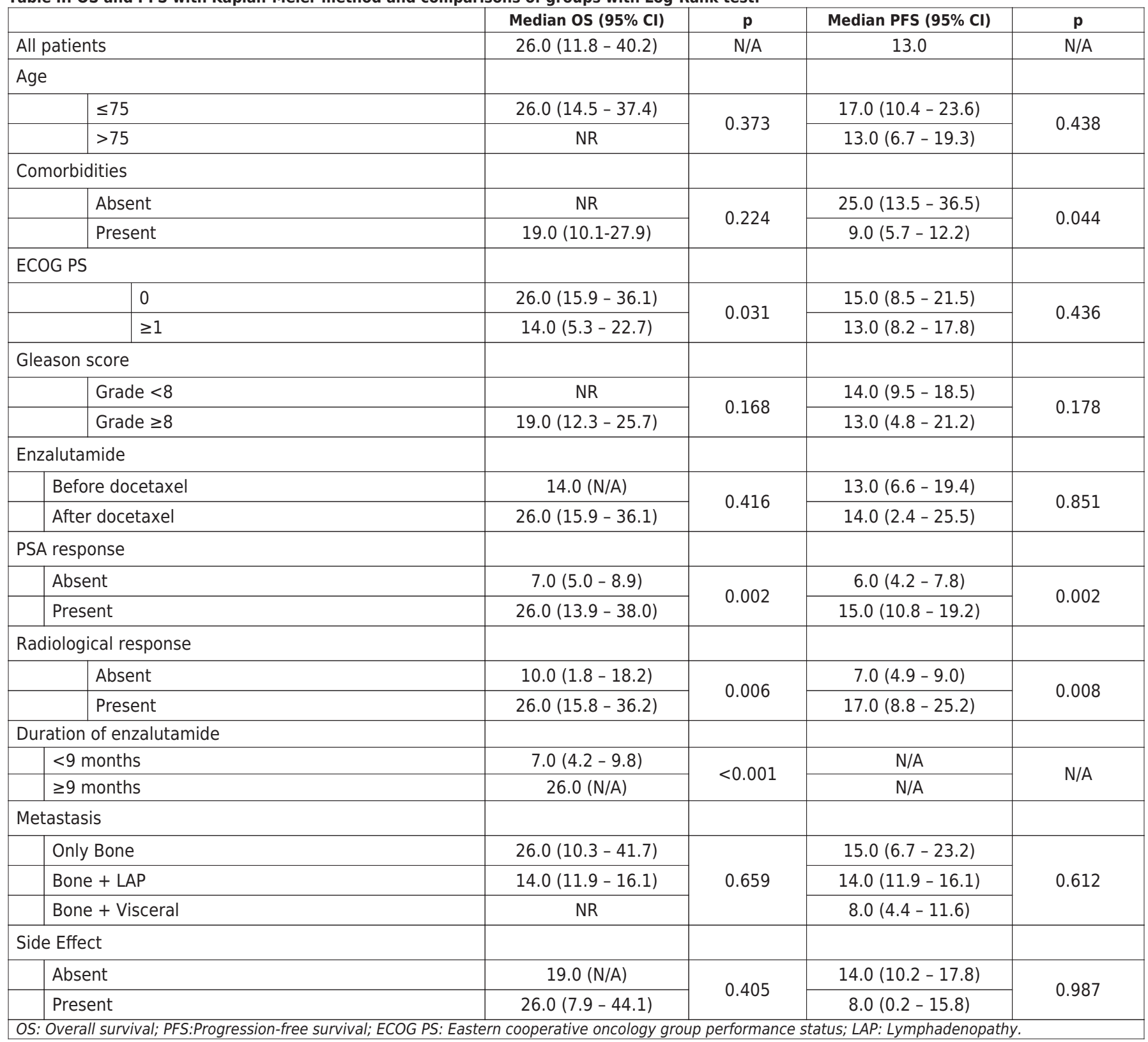

Upon intergroup comparisons of PFS by using the Log-Rank test, it was observed that PFS was significantly longer in those with PSA response (15.0 vs. 6.0 months, $p=0.002$, Figure 2), absence of comorbidities ( 25.0 vs. 9.0 months, $p=0.044)$ and radiological response (17.0 vs. 7.0 months, $p=0.008$, Table II).

It was determined that the presence of PSA response and radiological response to be excellent prognostic factors for PFS on Cox regression analysis. Patients with PSA response had 0.36 -fold lower risk of death [CI.95\% (0.16-0.85)]. Patients with PSA response had 0.38-fold lower risk of death [Cl.95\% (0.19-0.77)].

Side effects of any grade were observed in 32 patients $(47.8 \%)$, including fatigue in $28(41.8 \%)$, arthralgia in 21
(31.3\%), weight loss in $11(16.4 \%)$, and cerebrovascular event in $2(3 \%)$. No serious adverse events were noted that required discontinuation of treatment except for the cerebrovascular events (one patient with a subdural hematoma and one with encephalopathy owing to hypertension). No significant intergroup difference was observed regarding side effect frequency (Table I).

\section{DISCUSSION}

In this study, the efficacy and safety of enzalutamide in MCRPC before and after docetaxel was evaluated. Although the patients with poor performance score, older age and higher comorbidities given first-line enzalutamide, it was effective and tolerable treatment both in the first-line and in the second-line treatment of MCRPC. 


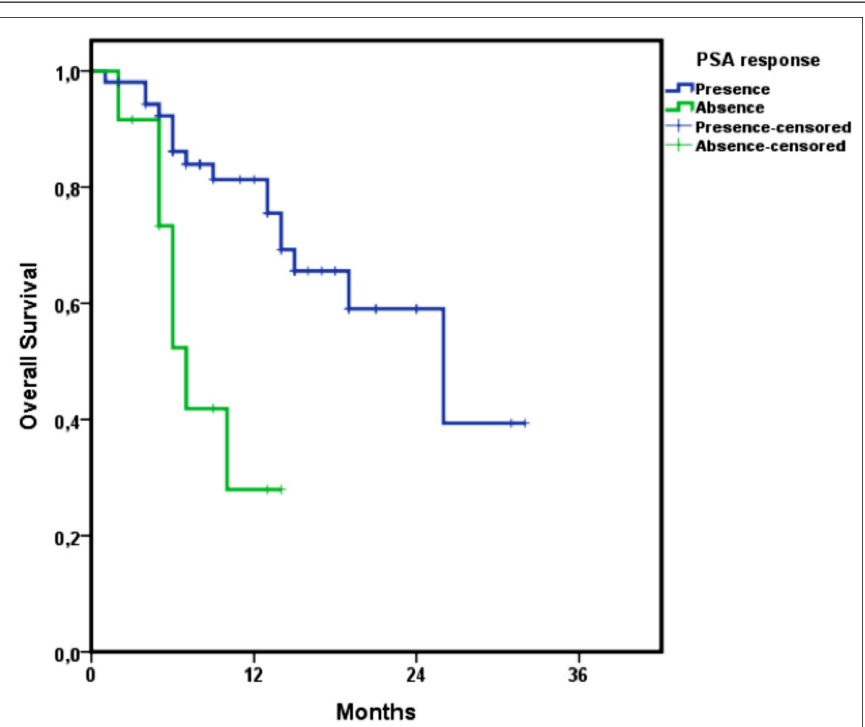

Figure 1: Overall survival Kaplan-Meier curve according to PSA response.

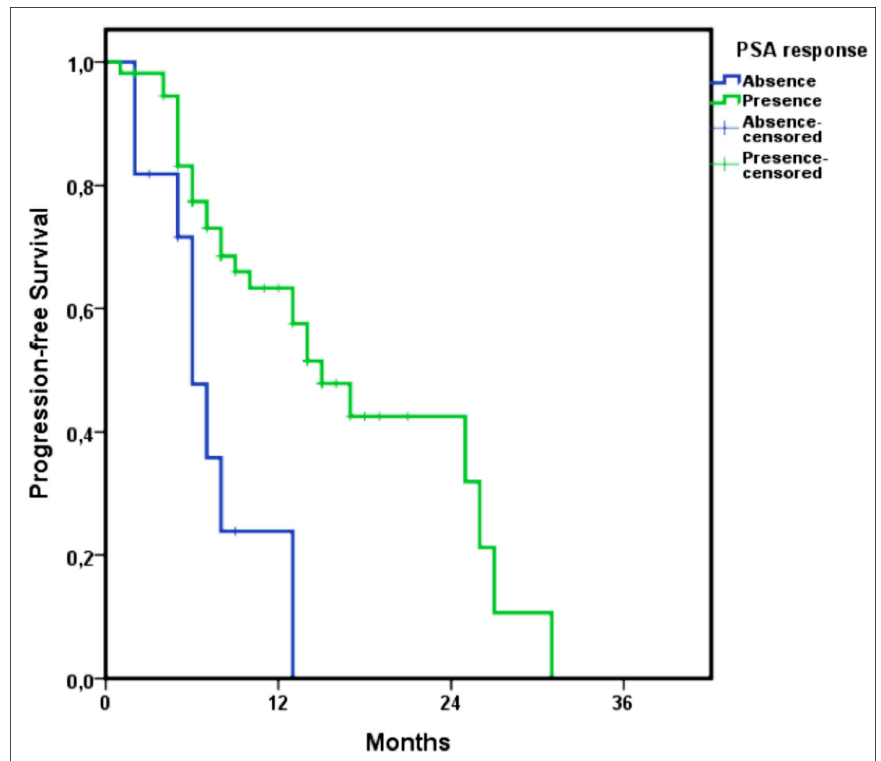

Figure 2: Progression-free survival Kaplan-Meier curve, according to PSA response.

This study determined a significantly higher OS and PFS in patients with PSA response. Moreover, higher than 9 months enzalutamide treatment duration was noted to decrease the risk of death. On the other hand, performance score $\geq 1$ (compared to ECOG PS 0), absence of PSA response and radiological response were determined to affect the survival adversely; notably, in patients with PSA and radiological response decreases the risk of progression.

In the present study, the PSA levels were observed to decrease significantly in all groups. PSA response was observed in 55 patients, 22 (78.6\%) of the BD group, and 33 (84.6\%) of the AD group. In the pivotal phase III PREVAIL study that used enzalutamide before chemotherapy form CRPC, PSA response was observed in $78 \%$ of patients, similar to the results of the present study. ${ }^{18}$ In another pivotal phase III AFFIRM study that used enzalutamide after chemotherapy for mCRPC, $54 \%$ of patients had PSA response; whereas, this rate was $84.6 \%$ in the $A D$ group in the current study. ${ }^{19}$ Upon examining the other studies in the literature that evaluated the effectiveness of enzalutamide treatment, PSA response rates in chemotherapy-naive patients were noted to vary between $43.2 \%-74.0 \%$; ${ }^{20-22}$ whereas, this ratio was approximately $25.4 \%-32.0 \%$ in docetaxel-pretreated patients. ${ }^{20,21}$ The reason for the considerably high PSA response rates in the AD group of current study compared with the literature could be because of the small and heterogeneous study group that was selected retrospectively. Moreover, the BD group was treated with enzalutamide because of their poor performance score (unsuitability for first-line docetaxel chemotherapy) and had more comorbidities compared with the AD group.

In Turkey, post-docetaxel enzalutamide treatment for $\mathrm{mCRPC}$ is reimbursed. Moreover, enzalutamide is administered to patients who cannot tolerate docetaxel (older age, poor ECOG PS) with the off-label approval of the Ministry of Health. According to the current study, the median OS in the BD group was 14 months, and that in the AD group was 26 months, with a no significant intergroup difference. Furthermore, longer enzalutamide treatments decreased the risk of death. In the PREVAIL study, the median OS with enzalutamide and placebo was 32.4 months and 30.2 months, respectively. Notably, enzalutamide provided a $29 \%$ reduction in the risk of death compared with placebo. ${ }^{18}$ The effect on OS might have been less than anticipated in current study because patients in the BD group were older and fragile, with a poor performance score. Nevertheless, three of the current study patients treated with enzalutamide as first-line owing to their poor performance scores were able to tolerate docetaxel treatment during progression.

The AFFIRM study revealed that enzalutamide significantly improved the median OS compared with placebo (18.4 vs. 13.6 months, HR $0.63, p<0.001) .{ }^{19}$ A prospective study on enzalutamide observed that the OS of the BD group was significantly longer than that of the AD group $(p=0.004) .^{{ }^{0}}$ Another retrospective study reported a median OS of 16.2 months for the BD group and 11.6 months for the $A D$ group with enzalutamide treatment $(p=0.003) .{ }^{21}$ Some studies have observed a trend of longer OS in patients with enzalutamide treatment before chemotherapy. ${ }^{22}$ However, results of the present study seem to be contradictory concerning the OS. Nevertheless, this contradiction could be explained, based on ethnic differences and patient characteristics (such as age and performance scores) of the study population.

The AFFIRM and PREVAIL studies determined that enzalutamide significantly increased the PFS compared with placebo. ${ }^{19}$ However, the literature lacks enough studies regarding the effect of enzalutamide, both $B D$ and $A D$, on the PFS. In the present study, the median PFS was numerically 
longer in the AD group (14 months) than the BD group (13 months), albeit with a negligible intergroup difference ( $p=$ 0.851). According to a multivariate analysis, PFS was significantly longer in patients with PSA response. Real-life data from Japan revealed that the median PFS was 12.9 months in the BD group and 7.3 months in the AD group, with a nonsignificant difference. ${ }^{20}$ The results of multivariate analysis from the same study revealed that an early PSA response of $50 \%$ was an independent predictor of better PFS. ${ }^{20}$ On the other hand, another study revealed that the effect of enzalutamide treatment on PFS was significantly higher in the BD group than in the AD group. ${ }^{21}$ In the present study if BD group was of younger age, good ECOG PS and had low comorbidities, the OS and PFS results would be better.

Approximately half of the current study patients $(47.8 \%)$ had side effects of any grade, including fatigue $(n=28,41.8 \%)$ and cerebrovascular event $(n=2,3 \%)$. In the PREVAIL study, $97 \%$ of patients had side effects of any grade, with the most common being fatigue (in 36\%), similar to the current study. Notably, the side effects of an acute coronary syndrome (1\%) and cerebrovascular event (1\%) were relatively low. ${ }^{18}$ In the AFFIRM study, 34\% of patients developed fatigue; whereas, $3 \%$ of patients developed side effects that caused death. ${ }^{19}$ The mismatch between the literature and the current results concerning side effect profiles might be because of the retrospective nature of this study.

Nonetheless, this study had some limitations. First, this study was conducted retrospectively with a relatively low number of patients. Second, the characteristics of treatment groups were heterogeneous. This heterogeneity was mainly because of the unavoidable fact that patients receiving enzalutamide as first-line treatment were those who were unable to tolerate chemotherapy, in parallel to Turkey's confirmations of the medicine.

Notably, in Turkey, enzalutamide is administered as a first-line treatment for MCRPC only in patients who cannot tolerate docetaxel. The present study is crucial because it provides real-world data reflecting the safety and reliability of enzalutamide in Turkey. Nevertheless, multicenter prospective studies are needed to analyse the sequencing of treatment form CRPC considering all the available treatment options.

\section{CONCLUSION}

This study revealed that enzalutamide is effective and safe for MCRPC, based on the real-world experience of both docetaxel-naive fragile patients and docetaxel-pretreated patients. PSA response, radiological response and duration of enzalutamide treatment may predict the improvement of survival.

\section{ETHICAL APPROVAL:}

Ethics Committee approval was received from the Ethics Committee of University of Health Sciences, Dr. Abdur- rahman Yurtaslan Oncology Training and Research Hospital (Number: E-75-30.07.2019).

\section{PATIENTS' CONSENT:}

Informed consents were obtained from all participants or their family, included in the study.

\section{CONFLICT OF INTEREST:}

Authors declared no conflict of interest.

\section{AUTHORS' CONTRIBUTION:}

IB: Conception and design, analysis and interpretation of data, drafting of the manuscript.

FBB: Analysis and interpretation of data.

HYC: Acquisition of data.

EE: Analysis and interpretation of data.

OB: Advices and final approval.

UD: Conception and design, reviewed the paper, and final approval.

\section{REFERENCES}

1. Attard G, Parker C, Eeles RA, Schröder F, Tomlins SA, Tannock I, et al. Prostate cancer. Lancet 2016; 387:70-82.

2. Siegel RL, Miller KD, Jemal A. Cancer statistics, 2018. CA Cancer J Clin 2018; 68:7-30.

3. Grossmann M, Cheung AS, Zajac JD. Androgens and prostate cancer; pathogenesis and deprivation therapy. Best Pract Res Clin Endocrinol Metab 2013; 27(4):603-16. doi: 10.1016/j.beem.2013.05.001.

4. Imamoglu GI, Tulay E, Ozdemir NY, Karacin C, Cimen S, Cilbir $\mathrm{E}$, et al. Do patient characteristics and duration of response to hormonal therapy predict outcomes of post-docetaxel abirateron acetate treatment in prostate cancer? UHOD. 2019; 29(3):001-8.

5. Sharifi N, Gulley JL, Dahut WL. Androgen deprivation therapy for prostate cancer. JAMA 2005; 294(2):238-44. doi:10.1001/jama.294.2.238.

6. Scher HI, Halabi S, Tannock I, Morris M, Sternberg CN, Carducci MA, et al. Design and end points of clinical trials for patients with progressive prostate cancer and castrate levels of testosterone: Recommendations of the prostate cancer clinical trials working group. J Clin Oncol 2008; 26(7):1148-59. doi: 10.1200/JCO.2007.12.4487.

7. Beaver JA, Kluetz PG, Pazdur R. Metastasis-free survival - a new end point in prostate cancer trials. N Engl J Med 2018; 378(26):2458-60. doi: 10.1056/NEJMp1805966

8. Loriot Y, Miller K, Sternberg CN, Fizazi K, De Bono JS, Chowdhury $\mathrm{S}$, et al. Effect of enzalutamide on health-related quality of life, pain, and skeletal-related events in asymptomatic and minimally symptomatic, chemotherapy-naive patients with metastatic castration-resistant prostate cancer (PREVAIL): Results from a randomised, phase 3 trial. Lancet Oncol 2015; 16(5):509-21. doi: 10.1016/S14702045(15)70113-0

9. Teo MY, Rathkopf DE, Kantoff P. Treatment of advanced prostate cancer. Annu Rev Med 2019; 70:479-99. doi: 10.1146/annurev-med-051517-011947. 
10. de Bono JS, Oudard S, Ozguroglu M, Hansen S, Machiels JP, Kocak I, et al. Prednisone plus cabazitaxel or mitoxantrone for metastatic castration-resistant prostate cancer progressing after docetaxel treatment: A randomised openlabel trial. Lancet 2010; 376(9747):1147-54. doi: 10.1016/S0140-6736(10)61389-X.

11. Knudsen KE, Scher HI. Starving the addiction: New opportunities for durable suppression of AR signaling in prostate cancer. Clin Cancer Res 2009; 15(15):4792-8. doi: 10.1158/1078-0432.CCR-08-2660

12. Montgomery RB, Mostaghel EA, Vessella R, Hess DL, Kalhorn TF, Higano CS, et al. Maintenance of intratumoral androgens in metastatic prostate cancer: A mechanism for castration-resistant tumor growth. Cancer Res 2008; 68(11):4447-54. doi: 10.1158/0008-5472.CAN-08-0249

13. Schalken J, Fitzpatrick JM. Enzalutamide: Targeting the androgen signalling pathway in metastatic castration-resistant prostate cancer. BJU Int 2016; 117(2):215-25. doi: 10.1111/bju.13123.

14. Dai C, Heemers H, Sharifi N. Androgen signaling in prostate cancer. Cold Spring Harb Perspect Med 2017; 7:a030452.

15. Ryan CJ, Smith MR, Fizazi K, Saad F, Mulders PF, Sternberg $\mathrm{CN}$, et al. Abiraterone acetate plus prednisone versus placebo plus prednisone in chemotherapy-naive men with metastatic castration-resistant prostate cancer (COUAA-302): Final overall survival analysis of a randomised, double-blind, placebo-controlled phase 3 study. Lancet Oncol 2015; 16(2):152-60. doi: 10.1016/S1470-2045 (14)71205-7.

16. Van Soest RJ, van Royen ME, de Morree ES, Moll JM, Teubel W, Wiemer EA, et al. Cross-resistance between taxanes and new hormonal agents abiraterone and enzalutamide may affect drug sequence choices in metastatic castration-resistant prostate cancer. Eur J Cancer 2013; 49(18): 3821-30. doi: 10.1016/j.ejca.2013.09.026.

17. Sanford M. Enzalutamide: A review of its use in metastatic, castration-resistant prostate cancer. Drugs 2013; 73(15):1723-32. doi: 10.1007/s40265-013-0129-9.

18. Beer TM, Armstrong AJ, Rathkopf DE, Loriot $Y$, Sternberg CN, Higano CS, et al. Enzalutamide in metastatic prostate cancer before chemotherapy. N Engl J Med 2014; 371(5):424-33. doi: 10.1056/NEJMoa1405095.

19. Scher HI, Fizazi K, Saad F, Taplin ME, Sternberg CN, Miller K, et al. Increased survival with enzalutamide in prostate cancer after chemotherapy. N Engl J Med 2012; 367(13): 1187-97. doi: 10.1056/NEJMoa1207506.

20. Kato H, Furuya Y, Miyazawa $Y$, Miyao T, Syuto T, Nomura M, et al. Consequences of an early PSA response to enzalutamide treatment for Japanese patients with metastatic castration-resistant prostate cancer. Anticancer Res 2016; 36(11):6141-9. doi: 10.21873/anticanres.11205.

21. Nadal R, Zhang Z, Rahman H, Schweizer MT, Denmeade SR, Paller CJ, et al. Clinical activity of enzalutamide in docetaxelnaïve and docetaxel-pretreated patients with metastatic castration-resistant prostate cancer. Prostate 2014; 74(15):1560-8. doi: 10.1002/pros.22874.

22. Jung SI, Kim MS, Jeong CW, Kwak C, Hong SK, Kang SH, et al. Enzalutamide in chemotherapy-naive patients with metastatic castration-resistant prostate cancer: A retrospective Korean multicoated study in a real-world setting. Investig Clin Urol 2020; 61: 19-27. 